\title{
A Study to Compare Antigen-based Enzyme-linked Immunosorbent Assay, Rapid Method, and Microscopy for Detection of Malaria in Blood Donors to prevent Transfusion-transmitted Malaria
}

\author{
${ }^{1}$ Manisha Shrivastava, ${ }^{2}$ Priya Nirankari, ${ }^{3}$ Sangita Singh, ${ }^{4}$ Seema Navaid
}

\begin{abstract}
Introduction: In malaria-endemic countries, blood donors with low parasitemia may be missed at the time of screening of blood units leading to the consequences like hemolysis, morbidity, and even mortality in nonimmune transfusion recipient. This study aimed to compare peripheral blood smear (microscopy), rapid diagnostic test (RDT), and antigen detection enzyme-linked immune sorbent assay (ELISA) for the detection of Plasmodium spp. in blood donors to prevent transfusiontransmitted malaria (TTM).
\end{abstract}

Materials and methods: Samples from 100 eligible blood donors coming to donate blood at the Bhopal Memorial Hospital \& Research Center blood bank were collected in ethylenediaminetetraacetic acid vial. Light microscopic examination on both thin and thick smears, RDT, and PAN-specific lactate dehydrogenase antigen detection ELISA were performed on the blood samples and the results were compared.

Results: None of the blood smears or samples from the 100 blood donors screened showed malarial parasitemia or positive result.

Conclusion: In India, the current strategy of testing blood donors is mainly restricted to microscopy and some blood banks have adapted rapid methods for antigen detection. A larger study on large sample size should be conducted to determine the most appropriate and effective malarial screening test in blood donors and, most importantly, to suggest a workable national screening policy for prevention of TTM in transfusion recipients.

Clinical significance: For screening blood units for malaria, currently available testing methodologies need to be studied in comparison with the assays with better sensitivity for increasing blood safety and reducing the complications of transmission of malaria to patients through blood transfusions.

Keywords: Antigen-based assays, Donor screening, Rapid diagnostic test, Transfusion-transmitted infections.

\footnotetext{
${ }^{1}$ Professor, ${ }^{2}$ Student, ${ }^{3}$ Laboratory Technician, ${ }^{4}$ Medical Officer

1,3,4 Department of Transfusion Medicine, Bhopal Memorial Hospital \& Research Center, Bhopal, Madhya Pradesh, India

${ }^{2}$ Department of Biotechnology, Awadhesh Pratap Singh University, Rewa, Madhya Pradesh, India

Corresponding Author: Manisha Shrivastava, Professor Department of Transfusion Medicine, Bhopal Memorial Hospital \& Research Center, Bhopal, Madhya Pradesh, India Phone: +917552742549, e-mail: manishasdr@gmail.com
}

How to cite this article: Shrivastava M, Nirankari P, Singh S, Navaid S. A Study to Compare Antigen-based Enzyme-linked Immunosorbent Assay, Rapid Method, and Microscopy for Detection of Malaria in Blood Donors to prevent Transfusiontransmitted Malaria. Int J Recent Surg Med Sci 2017;3(2):88-92.

Source of support: Nil

Conflict of interest: None

\section{INTRODUCTION}

Despite the fact that blood transfusions are essential to save lives of patients in emergency situations, there are associated risks, as many infectious diseases can be transmitted through blood transfusion. Malaria, hepatitis, syphilis, and acquired immune deficiency syndrome are recognized as major diseases transmitted through blood transfusion. Among the blood-borne infectious diseases, malaria is the oldest known and most common transfusion-transmitted infection (TTI). Malaria is an acute infectious, febrile and life-threatening disease caused by protozoan parasites from the genus Plasmodium. ${ }^{1}$ The prevalence of TTM varies from 0.2 cases per million in nonendemic countries to 50 or more cases per million in endemic countries. In malaria-endemic countries, blood donors with low parasitemia may be missed at the time of screening of blood units leading to the consequences like hemolysis, morbidity, and even mortality in nonimmune transfusion recipient. ${ }^{2}$ Blood banks and transfusion services are screening units and not diagnostic clinics and the purpose of screening blood units is to rule out donations that are at risk of transmitting infections. For selecting a test for detection of malaria, certain important factors like prevalence, sensitivity of the test, positive predictive value, assay reliability, speed, and cost need to be taken in to account. According to the World Health Organization $^{3}$ guidelines, the minimum evaluated sensitivity and specificity levels of all assays used for blood screening should be as high as possible and preferably not less than $99.5 \%$. But, the sensitivity of microscopy and rapid detection tests for malaria detection in blood units is much lower than the required, $\sim 50$ parasites $/ \mu \mathrm{L}$ diagnostic $\sim 100$ parasites $/ \mu \mathrm{L}$ respectively, while 1 to 10 parasites/ unit of blood or approximately 0.00004 parasites $/ \mu \mathrm{L}$ are 
enough to cause TTM. Moreover, in endemic countries, there could be loss of blood units donated by voluntary donors in an effort of detecting blood units with low parasitemia, and use of tests like polymerase chain reaction (PCR) could be cost prohibitive and are not routinely practiced. ${ }^{4}$ The modern techniques available for screening malaria are ELISA and PCR, which are not in routine practice in the developing world. In India, the current strategy of testing blood donors is mainly restricted to microscopy and some blood banks have adapted rapid methods for antigen detection. This pilot study aimed to compare antigen detection by ELISA with RDT and light microscopy for the detection of malaria in blood donors to prevent TTM.

\section{MATERIALS AND METHODS}

\section{Subject Selection}

A total of 100 normal healthy voluntary blood donors attending the blood bank of the Department of Transfusion Medicine were selected as subjects of the study. The study was done as part of a dissertation project and as ELISA was not routinely done for malaria screening, it was undertaken to standardize the process with a sample size of 100 due to financial constraints. Donors were selected according to donor screening criteria as per regulatory requirement of the Drugs and Cosmetics Act. History of fever within last 3 months and other questions specific to malaria were also included as a part of donor questionnaire. Samples for routine blood grouping and transfusion-transmitted disease testing of the donor unit are collected from donors selected for blood donation. The study was approved by the Institutional Ethical Committee (IEC), and for the additional tests for malaria detection, blood samples were collected from blood donors who gave consent for participation in this study after dully filling consent forms in a format as approved by the IEC.

\section{Methods}

\section{Microscopy}

Thick and thin peripheral blood smears were made from blood samples collected from blood donors coming to donate blood at a Department of Transfusion Medicine Blood Bank to detect the presence of malarial parasite with a microscope (Becton Dickinson). The thin blood smear was stained by using Giemsa stain to give an idea of the morphology of red cells and identity the stage of malaria parasite in its life cycle. The thick smear was stained by Field's stain, without fixing in methanol that removes the red cells to leave behind the imprints and pink parasite dots, if parasite is present.

\section{Rapid Diagnostic Test}

The RDT was done on blood samples by a kit (Lab Diagnostics) for the detection of $P$. falciparum-specific histidine rich protein-II (Pf HRP-II), malaria P. vivax, and $\mathrm{pLDH}$. The RDT was used to detect malaria antigen in blood flowing along a membrane containing specific antimalarial antibodies. The RDT device used in the study contained a membrane device precoated with two monoclonal antibodies as two separate lines across a test membrane. One monoclonal antibody (test line 1) was specific to the Pf HRP-II and another monoclonal antibody (test line 2) was specific to the pLDH of Plasmodium species (P. vivax, P. malaria, P. ovale). The HRP-II is a watersoluble protein produced by asexual stages and young gametocytes of $P$. falciparum and expressed on the RBC membrane surface. The $\mathrm{pLDH}$, an enzyme found in the glycolytic pathway of the malaria parasite, is produced by sexual and asexual stages of the parasite. Control Line " $\mathrm{C}$ " was precoated with goat antimouse antibody.

\section{Enzyme-linked Immunosorbent Assay}

Detection of malaria-specific antigen $(\mathrm{pLDH})$ in donor's blood was done by ELISA kit (Qualpro, Tulip Diagnostics) using ELISA Reader (Thermo Fisher Scientific, and Washer, Wellwash4MK2, Thermo Fisher Scientific). Wells of polystyrene microELISA were coated with monoclonal anti-pLDH antibodies. Another anti-pLDH antibody was biotinylated. Samples along with positive and negative controls were added in the coated wells and incubated simultaneously with biotinylated antibody. The wells were washed to remove unbound components. The presence of biotinylated antibodies was detected by adding streptavidin-horseradish peroxidase conjugate. After washing wells to remove unbound enzyme, substrate was added. The reaction was stopped after specified time with acid and absorbance was determined for each well at $450 \mathrm{~nm}$ with an ELISA reader.

\section{Interpretation of Result}

The cutoff value was calculated as per manufacturer's instructions by adding 0.1 to average absorbance value of negative control and absorbance of all the wells was compared with the cutoff value. Any sample having absorbance more than the cut off value was considered reactive. Sample with absorbance value less than the cutoff value was considered negative for malaria.

\section{RESULTS}

In this study, blood samples from 100 eligible blood donors (99 males and 1 female) were tested for malaria using thick and thin blood smears, rapid test, and antigen 
Table 1: Malaria parasitemia with respect to age among blood donors

\begin{tabular}{lll}
\hline & \multicolumn{2}{c}{ Total no. of blood donors } \\
\cline { 2 - 3 } Age groups (in years) & $\begin{array}{l}\text { No. of candidates } \\
\text { examined }\end{array}$ & $\begin{array}{l}\text { No. of infected } \\
\text { candidates }\end{array}$ \\
\hline $18-22$ & 35 & 0 \\
$23-27$ & 28 & 0 \\
$28-32$ & 20 & 0 \\
$33-37$ & 5 & 0 \\
$38-42$ & 8 & 0 \\
$43-47$ & 1 & 0 \\
$48-52$ & 1 & 0 \\
$53-57$ & 0 & 0 \\
$58-62$ & 1 & 0 \\
Total & 100 & 0 \\
\hline
\end{tabular}

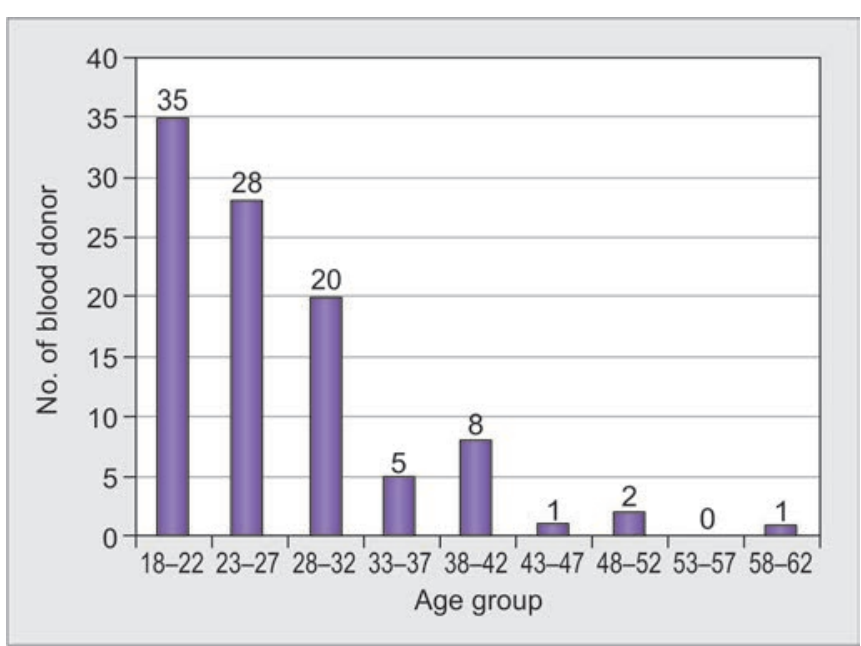

Graph 1: Age group-wise distribution of blood donors

Table 2: Results of ELISA on blood donor samples

\begin{tabular}{|c|c|c|c|c|c|c|}
\hline Batch no. & $\begin{array}{l}\text { Total no. } \\
\text { of samples } \\
\text { included }\end{array}$ & $\begin{array}{l}\text { No. of } \\
\text { positive } \\
\text { controls }\end{array}$ & $\begin{array}{l}\text { No. of } \\
\text { negative } \\
\text { controls }\end{array}$ & $\begin{array}{l}\text { Mean value of } \\
\text { OD for positive } \\
\text { control }\end{array}$ & $\begin{array}{l}\text { Mean value of } \\
\text { OD for negative } \\
\text { control }\end{array}$ & $\begin{array}{l}\text { Mean value } \\
\text { of OD for test } \\
\text { samples }\end{array}$ \\
\hline 1 & 28 & 2 & 2 & 1.287 & 0.012 & 0.112 \\
\hline 2 & 23 & 2 & 3 & 1.423 & 0.014 & 0.114 \\
\hline 3 & 27 & 2 & 3 & 1.548 & 0.020 & 0.120 \\
\hline 4 & 22 & 2 & 2 & 1.901 & 0.005 & 0.105 \\
\hline
\end{tabular}

detection-based ELISA. Most donors (83\%) were young, between the age of 18 and 32 years (Table 1 and Graph 1 ). None of the blood smears from the 100 blood donors screened by Giemsa and field stain showed malarial parasitemia. The RDTdevice showed only control band (C) during the test of all 100 blood samples and thus, was considered negative for malaria. The pLDH antigen detection ELISA test for 100 blood samples was carried out in four batches. The cutoff values of optical density (OD) for sample was less than the cut off value of mean OD for positive control; hence, all batches and samples were found to be negative for malaria (Table 2).

In first batch, two positive controls and two negative controls were used, and their mean OD was 1.287 and 0.012 respectively. All the 28 samples tested in first batch had OD less than cutoff value, which was 0.112. All the 28 samples were interpreted as nonreactive for malaria. In the second batch, two positive controls and three negative controls were used and their mean OD was 1.423 and 0.014 respectively. All the 23 samples tested in second batch had OD less than cutoff value as 0.114 . All the 23 samples were nonreactive for malaria. In the third batch, two positive controls and three negative controls were used and their mean OD was 1.548 and 0.020 respectively. All the 27 samples tested in third batch had OD less than cutoff value, which was 0.120 . All the 27 samples were nonreactive for malaria. In fourth batch, two positive controls and two negative controls were used and their OD was
1.901 and 0.005 respectively. All the 22 samples tested in fourth batch had OD less than cutoff value, which is 0.105 and were nonreactive for malaria. The OD of each sample is represented in Graph 2. Thus, none of the blood donors were found to be positive for malaria by the three methods implied in this study including ELISA.

\section{DISCUSSION}

In endemic countries, screening by donor questionnaires with inclusion of questions on history of fever with in a stipulated time before donation ( 3 months in our country) is not sufficient as many blood donors carrying Plasmodium may be asymptomatic. For prevention of TTM, an effective and sensitive test is required for screening malaria in blood donors. In India, currently, microscopy and rapid tests are used for testing for malaria in blood donors. Most of the blood banks are using "thick and thin smear microscopy" as it is the gold standard and inexpensive for the diagnosis of malaria. Screening of malaria by microscopy was time consuming, requires manpower, and is not feasible while also being inadequate for examining large volumes of samples in blood banks. Also, it is far too insensitive to be recommended as the usable screening test for transfusion services in endemic countries.

The RDTs have a similar sensitivity to microscopic evaluation, although their sensitivity decreases when parasite load is smaller than 100 parasites $/ \mu \mathrm{L}$. The RDT 


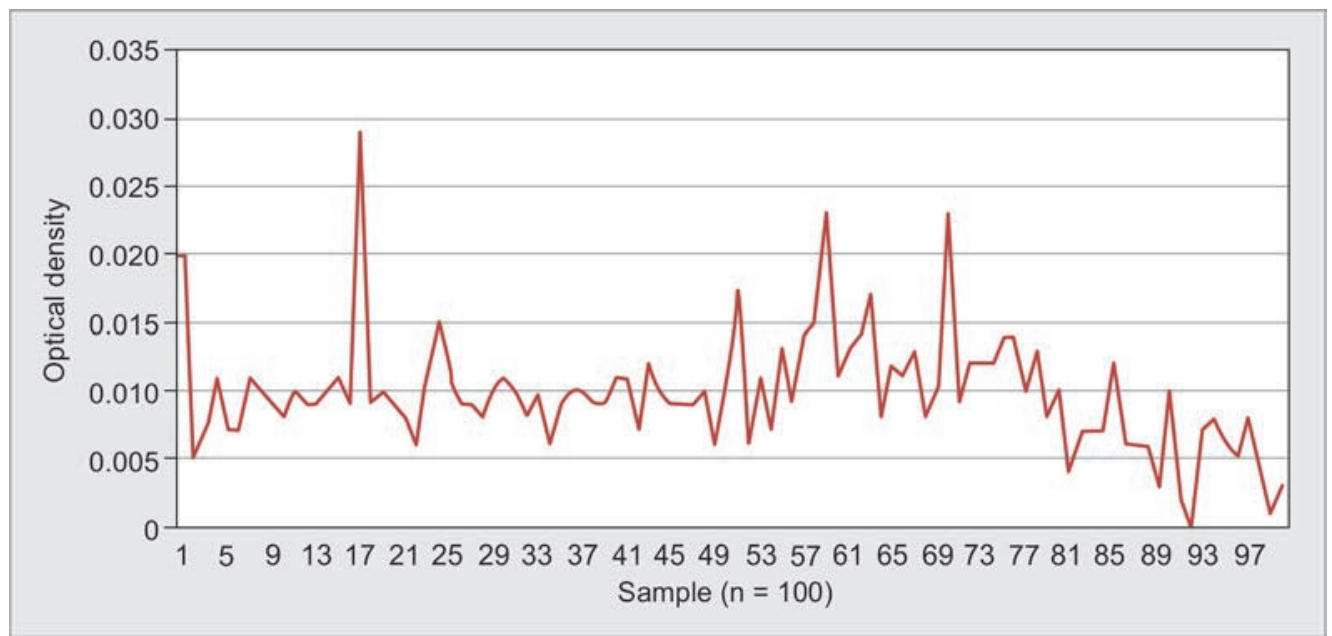

Graph 2: Distribution of OD in blood samples for malaria ELISA

was better than microscopy in terms of objectivity, and less stringent requirement for training than ELISA or PCR. In a study while the microscopic examination did not reveal malaria positivity, 2 out of 700 donors were detected as malaria positive on use of rapid test based on immunochromatography. ${ }^{5}$ In a scientific communication, authors recommended that use of peripheral smear screening of positive cases as gold standard with routine screening of blood donors with rapid detection devices as a reasonably reliable method to prevent TTM in India. ${ }^{6}$ The antigen detection ELISA has high sensitivity and takes about 2 to 2.5 hours to complete and can process batches of 50 to 100 samples simultaneously. A study that concluded that pLDH antigen ELISA could be an interesting tool for blood donation qualification in order to ensure blood safety in malaria-endemic areas. ${ }^{7}$ The ELISA is adequate for examining large volumes of sample in less time and is cost-effective. In our study, none of the blood donors were found to be positive for malaria by the three methods implied, and no differences in sensitivity or specificity of the three methods could be assessed as our findings are limited by small sample size. The prevalence of malaria may vary from 0.2 cases per million recipients in nonendemic countries to 50 or more cases per million in endemic countries. ${ }^{8}$ Studies show low prevalence of 0.2 and $0.009 \%$ respectively, of transfusion-transmitted malaria., ${ }^{910}$ Most of the malarianonendemic countries follow the rule of donor deferral for 3 years after malaria infection. The disadvantage of malarial antibody test is that a negative result does not guarantee that the donor is not infected with malarial parasites and in the initial few days of illness, the antibodies may not be detectable. The P. falciparum and $P$. vivax antigen-based assays may not detect infection with P. ovale and P. malariae. ${ }^{11}$ In endemic countries, screening for specific anti malarial antibody provides an effective means of minimizing the risk of transmission; however, in endemic countries, since the prevalence of malarial antibody is high in donor population, it would not be a feasible option as a lot of units will be discarded due to positive results. This study also suggested an option of screening with antibody detection assays, and positive units could be treated for pathogen inactivation. The authors suggested that the most ideal would be screening blood donation by PCR-based assays for detection of low parasitemia in order to prevent TTM. ${ }^{12}$ Another study, where only microscopy was used, advocated the use of ELISA and PCR for malaria screening of donated units in endemic countries. ${ }^{13}$ A review from sub-Saharan Africa concluded that in absence of suitable, affordable, and practical tests for malaria screening in blood donors and lack of clinical evidence options like pretransfusion, malaria prophylaxis may be considered in patients. The menace of malaria is high in endemic countries and it may not possible like other TTIs to completely prevent TTM. ${ }^{2}$ For a country like India, where antibody-endemic persons will only be potential blood donors, seeking history and rejecting the donors with history of fever within last 3 months is a feasible solution as done in our study. Our study used an ELISA based on antigen detection, which will be much useful and more studies could be done with a larger sample size. The sensitivity of the assay holds importance. The scope of this study and the results could not be conclusive due to the small sample size taken due to budget and time constraints. However, another study has been planned and the role of antigen ELISA along with PCR for malaria shall be studied with larger sample size. In addition, as per the criteria of donor selection, the possibility of getting malaria positive donors was rare, so no significant differences in sensitivity or specificity were found.

Malaria diagnoses in blood donors and patients are different in terms of objectives. Donor selection is based on being able to rule out donors with risk of transmitting 
malaria to transfusion recipients and the need is to use a rapid and robust method, while testing for malaria in clinics is based on test accuracy. The important determinants will be the malaria prevalence of the region and malaria immunity in the donor population along with the test sensitivity, cost, reliability, speed, and complexity. ${ }^{14}$

\section{CONCLUSION}

Though the prevalence of TTM is low, malaria due to blood transfusion may still occur despite predonation history taking and screening of blood donors. Any patient with febrile illness after blood transfusion may be a case of TTM and may also develop complications. There is an urgent need of multicentric nationally funded study in order to develop a national policy for addition of a better screening assay for malaria screening among blood donors for prevention of TTM, which could lead to serious posttransfusion problems in patients simulating a hemolytic transfusion reaction. The new methods for postdonation testing of blood units together with pretransfusion prophylaxis of malaria in highly endemic areas would ensure blood safety without affecting availability of blood.

\section{ACKNOWLEDGMENTS}

The authors thank the administrators of the institute for support.

\section{REFERENCES}

1. Torres KL, Moresco MNDS, Sales LR, Abranches JDS, Alexandre MAAA, Malheiro A. Transfusion-transmitted malaria in endemic zone: epidemiological profile of blood donors at the Fundacao HEMOAM and use of rapid diagnostic tests for malaria screening in Manaus. Rev Bras Hematol Hemoter 2014 Jul-Aug;36(4):269-274.

2. Nansseu JRN, Noubiap JJ, Ndoula ST, Zeh AFM, Monamele CG. What is the best strategy for the prevention of transfusion-transmitted malaria in sub-Saharan African countries where malaria is endemic? Malar J 2013 Dec;12:465.

3. World Health Organization. Screening donated blood for transfusion transmissible infections: recommendations. Geneva: World Health Organization; 2010.

4. Malik, V. Drug and cosmetics act of India 1940. 13th ed. Lucknow (India): Eastern Book Company; 2001.

5. Lakshmi S, Anuradha B. Prevalence of malaria in blood donors and risk of transfusion transmissible malaria. Int J Curr Microbiol App Sci 2015;4(8):352-357.

6. Bahadur S, Pujani M, Jain M. Use of rapid detection tests to prevent transfusion-transmitted malaria in India. Asian J Transfus Sci 2010 Jul;4(2):140-141.

7. Atchade PS, Lang CD, Chabi N, Perrotey S, Abdelrahman T, Akpovi CD, Anani L, Bigot A, Sanni A, Candolfi E. Is a Plasmodium lactate dehydrogenase (pLDH) enzyme-linked immunosorbent (ELISA)-based assay a valid tool for detecting risky malaria blood donations in Africa? Malar J 2013 Aug;12:279.

8. Rajab JA, Waithaka PM, Orinda DA, Scott CS. Analysis of cost and effectiveness of post transfusion screening of donor blood and antimalarial prophylaxis for recipients. East Afr Med J 2005 Nov;82(11):565-571.

9. Chandra T, RizviS NF, Agarwal D. Decreasing prevalence of transfusion transmitted infection in Indian scenario. Sci World J 2014 Jan;2014(2014):173939.

10. Shahtaj K, Farah F, Noor R, Fazle R. Reducing the risk of transfusion transmitted infections through predonation screening of blood donors. Int J Pathol 2011;9(2):59-62.

11. Kitchen A, Mijovic A, Hewitt P. Transfusion-transmitted malaria: current donor selection guidelines are not sufficient. Vox Sang 2005 Mar;88(3):200-201.

12. Dubey A, Elhence P, Ghoshal U, Verma A. Seroprevalence of malaria in blood donors and multi-transfused patients in Northern India: relevance to prevention of transfusiontransmissible malaria. Asian J Transfus Sci 2012 Jul-Dec; 6(2):174-178.

13. Ali N, Ahmed J, Ali N, Jehan F, Saleem S. Transfusion transmitted malaria in three major blood banks of Peshawar, Pakistan. Afr J Biotechnol 2010 Sep;9(33):5445-5449.

14. Nam MH, Kim JS, Cho CH, Han ET, Lee WJ, Lee HK, An SS, Lim CS, Lee KN. Evaluation of plasmodium vivax ELISA for the blood screen. Trop Med Int Health 2010 Dec;15(12): 1436-1441. 